\title{
Quantitative ABCD Analysis of Online Food Delivery Services
}

\author{
Dsouza Prima Frederick ${ }^{1} \&$ Ganesh Bhat S. $^{2}$ \\ ${ }^{1}$ Research Scholar, College of Management \& Commerce, Srinivas University, Mangalore- \\ 575001, India \\ OrcidID: 0000-0003-2568-5619; Email ID: primadsouza.cmc@ srinivasuniversity.edu.in \\ ${ }^{2}$ Research Professor, College of Management \& Commerce, Srinivas University, Mangalore, \\ India \\ OrcidID: 0000-0003-1950-8536; Email ID: ganbhatbvr@rediffmail.com
}

Area of the Paper: Business Management.

Type of the Paper: Research Analysis.

Type of Review: Peer Reviewed as per $|\mathrm{C}| \mathrm{O}|\mathrm{P}| \mathrm{E} \mid$ guidance.

Indexed In: OpenAIRE.

DOI: https://doi.org/10.5281/zenodo.6052817

Google Scholar Citation: IJCSBE

\section{How to Cite this Paper:}

Dsouza, Prima Frederick, \& Ganesh Bhat, S., (2022). Quantitative ABCD Analysis of Online Food Delivery Services. International Journal of Case Studies in Business, IT, and Education (IJCSBE), 6(1), 76-92. DOI: https://doi.org/10.5281/zenodo.6052817

International Journal of Case Studies in Business, IT and Education (IJCSBE)

A Refereed International Journal of Srinivas University, India.

Crossref DOI : https://doi.org/10.47992/IJCSBE.2581.6942.0152

(C) With Authors.

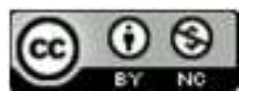

This work is licensed under a Creative Commons Attribution Non-Commercial 4.0 International License subject to proper citation to the publication source of the work.

Disclaimer: The scholarly papers as reviewed and published by the Srinivas Publications (S.P.), India are the views and opinions of their respective authors and are not the views or opinions of the S.P. The S.P. disclaims of any harm or loss caused due to the published content to any party. 


\title{
Quantitative ABCD Analysis of Online Food Delivery Services
}

\author{
Dsouza Prima Frederick ${ }^{1}$ \& Ganesh Bhat S. $^{2}$ \\ ${ }^{1}$ Research Scholar, College of Management \& Commerce, Srinivas University, Mangalore- \\ 575001, India \\ OrcidID: 0000-0003-2568-5619; Email ID: primadsouza.cmc@ srinivasuniversity.edu.in \\ ${ }^{2}$ Research Professor, College of Management \& Commerce, Srinivas University, Mangalore, \\ India \\ OrcidID: 0000-0003-1950-8536; Email ID: ganbhatbvr@rediffmail.com
}

\begin{abstract}
Purpose: The core idea of the study is to understand the development of $A B C D$ framework for analysing Online Food Delivery Services and to identify and evaluate the determinant issues and key attributes affecting the factors such as Advantages, Benefits, Constraints, and Disadvantages from stakeholders ' point of view and to find the major affecting factor in respect to Online Food Delivery Services.

Design: The study has systematically reviewed the published paper related to the $A B C D$ analysis framework from 2016 to 2021. The study employed quantitative analysis through focus group interaction by allotting appropriate weightage to key attributes to find the major affecting factors and critical constituent elements for Online Food Delivery Services.

Findings: $A B C D$ analysis framework is the technique used to analyse and evaluate a business model, concept, product, strategy or system. This technique is used by many researchers in different fields for the analysis of their research studies. Quantitative ABCD analysis of Online Food Delivery Services found that Advantages factors affect majorly for the success of the business. The result of ABCD analysis supports the concept/ideas effectiveness of Online Food Delivery Services.

Originality: The paper gives insights on the determinant issues, key attributes, major affecting factors and critical constituent component elements as per the ABCD analysis framework for Online Food Delivery Services.
\end{abstract}

Type of Paper: Empirical Analysis

Keywords: ABCD analysis framework, Online Food delivery Services, Quantitative ABCD analysis, Determinant issues, Key attributes, Factor Analysis, Elementary Analysis

\section{INTRODUCTION :}

The global expansion of e-commerce is being driven by economic prosperity and result of greater internet accessibility. Of late, consumers' disposable income has increased, e-payments have become safer, and the number of internet service providers has increased many folds, combined with the expansion and of their delivery services and reach, therefore, consumers are attracted to switch over to online services [1].

Digitalization, on the other hand, is a mega-trend that is rapidly being embraced by businesses that are attempting to innovate to capitalize their potential to the fullest extent [2]. Owing to this advancement, Online Food Delivery (OFD) services are becoming very popular in the e-commerce world. Moreover, in this twenty-first century more customers are drawn to a product or service online and then persuaded to buy it [3].

The development of OFD has revolutionized the human mind-set; many customers and food providers connect all over the world, as well as the industry in terms of sustainability impact (which are outlined by three variables: Social, Economic, and environmental issues) to define the extent for further advancements [4][5].

$\mathrm{ABCD}$ analysis framework has become a very popular technique used for analysis in recent years. Many researchers are using this framework to analyze their business model as it helps in identifying 
determinant issues, key attributes, and critical component elements, and also to know the affecting factors for the success of the business. This paper studies the background of the ABCD model development and the quantitative $\mathrm{ABCD}$ analysis is employed to evaluate the online food delivery services and find major affecting factor for the study.

\section{LITERATURE REVIEW ON ABCD FRAMEWORK :}

A business model is a collection of distinct processes or activities that lead the business to long term profit path through customer value creation. It explains the organization's strategy for continued growth [6]. Thus, a business model consists of an operation or a methodology where only the most crucial components of the business activity under discussion are highlighted.

Recently, Aithal, et al., (2016) developed a framework named ABCD which is used to analyse the different business models/concepts and which can also be used to understand its impacting factors in providing value to its various stakeholders or determinant issues and thereby, helps in analysing future income a business could earn for its stable growth. This framework is a unique analytical method for studying a business model, company strategy, concept, idea, or business system. This method generates a structured matrix including an outline of several business constructs abbreviated as ABCD, named as Advantages, Benefits, Constraints, and Disadvantages. This framework, thus, applied can give a fruitful outcome in a well-organized list of Advantages, Benefits, Constraints, and Disadvantages of determinant issues and various key attributes behind the determinant issues impacting the business/concept and within which each determinant issue is having critical component elements [7]. Moreover, this framework being simple by nature, provides effective guidelines to identify and analyse the effectiveness of any business model or operational concept. Several studies based on this framework have attempted to quantify the determinant issues and key attributes to calculate the scores based on ranks. In addition, weightage to the critical constituent elements are allotted and incorporated like other popular techniques- SWOC, Competitive Profile Matrix (CPM) analysis, EFE \& IFE Matrices, BCG matrix, Porter's Five Forces Model, and PESTLE Analysis [6], [8]-[11].

Furthermore, 'ABCD Analysis Technique' is being employed in the research studies "Working from Home" e-business model. The study analysed the factors affecting the work from home system using the ABCD model. It is observed from the analysis that factors affecting work from home were more advantageous and beneficial in comparison to constraints and disadvantages. Therefore, the WorkFrom-Home (WFH) model became more crucial from employers' and employees' point of view for the success of the organization [12] [13].

$\mathrm{ABCD}$ analysis framework is also used in a research paper on the concept- "Higher Education Stage Model". The features of the research study were determined based on identifying the issues affecting and analysing the Advantages, Benefits, Constraints, and Disadvantages. The findings of the study, supported the model ABCD [14].

In this hindsight, this paper has made an attempt to analyse and evaluate Online food Delivery Services by employing $\mathrm{ABCD}$ analysis framework. The affecting factors of Online Food Delivery Services is assessed based on identifying and analysing the Advantages, Benefits, Constraints, and Disadvantages under the determinant issues like Supplier Issues, Food deliverer Issues, Customer Issues, and Food Delivery Technology Issues. The various determinant issues and key attributes are identified using the $\mathrm{ABCD}$ analysis framework and the constituent critical element is identified for each factor using the focus group method.

\section{OBJECTIVES OF THE STUDY :}

The present research study has the following objectives:

(1) To discuss the suitability of ABCD analysis framework to analyse Online Food Delivery Services.

(2) To list Advantages, Benefits, Constraints, and Disadvantages of Online Food Delivery Services.

(3) To identify various determinant issues in respect of Online Food Delivery Services.

(4) To determine affecting factors under each key attribute as per ABCD analysis framework.

(5) To analyse the identified critical constituent element for each ABCD construct using its elementary analysis technique.

(6) To evaluate the critical constituent elements for each ABCD construct. 


\section{ABCD LISTING OF ONLINE FOOD DELIVERY SERVICES :}

The ABCD listing of online food delivery services are as follows:

\section{Advantages:}

The Online Food Delivery Services has the following advantages:

1. Entire order processing is hastened for desired food items.

2. The business can have an easier order management process in the maintenance of all records.

3. Cash registers need not be maintained as all the transaction records related to incomes and expenses can be retrieved from the ordering system.

4. Promotion costs can be reduced and expensive advertisement and sales promotion exercises can be avoided due to the presence of an online app in which all updates can be uploaded and the same can be available to the user all the time.

5. Customer database can be retrieved and effective customer retention strategies can be designed to retain the existing customers from switching to another supplier.

6. Customers get food items at reasonable price as the food supplier enjoys economy of bulk orders.

7. Updates on order can be easily tracked by the customer through food delivery apps.

8. Safe delivery is ensured to the customer by the supplier by following hygiene practices in food preparation.

9. Healthy food is ensured to the customer by use of fresh ingredients.

10. Easy user-friendly interface is provided to make the ordering process very easy.

\section{Benefits:}

The Online Food Delivery Services offer the following benefits:

1. The whole process of ordering provides the convenience of place and time for customers.

2. Increase in turnover of inventory and sales can be achieved with the outreach to many customers at a time.

3. The offer of food products by many vendors creates interest and desire among the customers to use the service apps to order food, therefore, it widens the customer base.

4. Many sales promotions like discounts, promo-codes, and coupons are offered to the customers, therefore, the customer is motivated to repurchase.

5. Choice and selection of food items at reasonable rates help the customers to get their desired orders from many restaurants at the same time to arrive at a purchase decision.

6. Supplier can enjoy economic order quantity benefits due to receipt of bulk orders.

7. Information and instructions displayed on website and apps are well presented to ease the process of food orders.

8. Customization on food orders, help supplier to deliver desired food orders.

9. Availability of many modes of cash and e-payments helps customer to select an option as per his/her convenience.

10. Eco-friendly packaging by the supplier helps in nature conservation.

\section{Constraints:}

Online Food Delivery Services face the following constraints:

1. Protection of the personal data of the customer/user from hacking or cyber-attacks is very difficult.

2. Changes in Government policies impacts the suppliers to modify their business operations.

3. Many competitors in the same business can imitate the business model, therefore, affecting the survival of the business.

4. Customer loyalty and retention are very challenging, as a disappointed customer may switch to another supplier.

5. Reduction of lead time in the process of food preparation and on time delivery is difficult.

\section{Disadvantages:}

The Online Food Delivery Services have the following disadvantages:

1. Stiff competition by rivals may limit the growth of business app.

2. Prices for food items cannot be fixed due to volatility in the market price of food ingredients. 
3. Insufficient training to the personnel involved in the delivery process can reduce the standard of delivery.

4. Outreach in remote areas or rural zones is limited due to the less- disposable income of the many rural residents.

5. Varied types of risks are faced such as food-associated risks, psychological risks, and efficiency risks for the parties involved in the whole process of delivery.

\section{ABCD FRAMEWORK FOR ONLINE FOOD DELIVERY SERVICES :}

Table 1: Exhibits of determinant issues identified by different authors in the various field by using $\mathrm{ABCD}$ analysis framework

\begin{tabular}{|c|c|c|c|}
\hline $\begin{array}{l}\text { Sl. } \\
\text { No }\end{array}$ & Area & Determinant Issues & $\begin{array}{l}\text { Reference } \\
\text { Number }\end{array}$ \\
\hline 1. & Black Ocean Strategy & $\begin{array}{l}\text { Organizational, Administrative, } \\
\text { Operational, Business, External and Operational }\end{array}$ & {$[8],[15]$} \\
\hline 2. & Accreditation & $\begin{array}{l}\text { Organizational, Faculty Performance, Student } \\
\text { Development/Progression, } \\
\text { Social/Environmental/Community Engagement }\end{array}$ & [9] \\
\hline 3. & $\begin{array}{l}\text { Private } \\
\text { Analysis }\end{array}$ & $\begin{array}{l}\text { Organisational, Student Progression, Faculty } \\
\text { Development, Societal and Other Stakeholders, } \\
\text { Governance, Innovation and Best Practices }\end{array}$ & {$[14],[16]$} \\
\hline 4. & $\begin{array}{ll}\text { New } & \text { National } \\
\text { Institutional } & \text { Ranking } \\
\text { System } & \end{array}$ & $\begin{array}{l}\text { Teaching And Learning Resources, Research } \\
\text { Productivity, Impact And IPR, Graduation } \\
\text { Outcome, Outreach and Inclusivity, Perception }\end{array}$ & {$[11]$} \\
\hline 5. & $\begin{array}{l}\text { Ideal Software and Its } \\
\text { Realization Scenarios }\end{array}$ & $\begin{array}{l}\text { Operational, Transitional, Maintenance, Output, } \\
\text { Environment }\end{array}$ & [17] \\
\hline 6. & $\begin{array}{ll}\text { Annual } & \text { Research } \\
\text { Productivity } & \\
\end{array}$ & $\begin{array}{l}\text { Organizational, Academic \& Curriculum, Faculty, } \\
\text { Students, Other Stakeholders }\end{array}$ & [18] \\
\hline 7. & $\begin{array}{l}\text { Dye-Doped Polymers for } \\
\text { Photonic Applications }\end{array}$ & 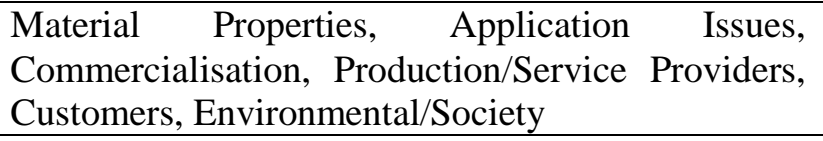 & [19] \\
\hline 8. & $\begin{array}{l}\text { Factors \& Elemental } \\
\text { Analysis of Six Thinking } \\
\text { Hats Technique }\end{array}$ & $\begin{array}{l}\text { Conceptual, Managerial, } \quad \text { Operational, } \\
\text { Organisational, Societal, and Stakeholder }\end{array}$ & [20] \\
\hline 9. & $\begin{array}{l}\text { Factor Analysis Based on } \\
\text { ABCD Framework on } \\
\text { Recently Announced } \\
\text { New Research Indices }\end{array}$ & $\begin{array}{l}\text { Research Organisation, Researcher, Funding } \\
\text { Agency, Industry }\end{array}$ & [21] \\
\hline 10. & $\begin{array}{lc}\text { Task } & \text { Shifting- } \\
\text { Professional } & \text { Healthcare } \\
\text { Personnel Shortage }\end{array}$ & $\begin{array}{l}\text { Organizational, Alternative Acceptors, Donor } \\
\text { Physicians, Patients \& Relative, Societal, Country }\end{array}$ & [22] \\
\hline 11. & $\begin{array}{l}\text { IEDRA Model of } \\
\text { Placement Determination }\end{array}$ & $\begin{array}{l}\text { Model Schedule, Model Flexibility, Model } \\
\text { Administration, Overall Model Relevance and } \\
\text { Applicability }\end{array}$ & [23] \\
\hline 12. & $\begin{array}{l}\text { Generating Wealth at the } \\
\text { Base of the Pyramid }\end{array}$ & $\begin{array}{l}\text { Stakeholder, Business, Operational, Customer, } \\
\text { Technological and Environment \& Social }\end{array}$ & [24] \\
\hline
\end{tabular}

Table 2: Exhibits of scholarly contribution by different authors in the various field by using ABCD analysis framework

\begin{tabular}{|c|c|c|c|}
\hline $\begin{array}{l}\text { Sl. } \\
\text { No. }\end{array}$ & Area & Findings & $\begin{array}{l}\text { Reference } \\
\text { Number }\end{array}$ \\
\hline 1. & $\begin{array}{l}\text { Analysis of Choice } \\
\text { Based Credit } \\
\text { System in Higher } \\
\text { Education }\end{array}$ & $\begin{array}{l}\text { SWOC and ABCD analysis were used in the study which } \\
\text { aimed to perform a comparative analysis of the "Choice } \\
\text { Based Credit System." The study found CBCS provides } \\
\text { students with simple migration to a wide range of }\end{array}$ & [25] \\
\hline
\end{tabular}




\begin{tabular}{|c|c|c|c|}
\hline & & $\begin{array}{l}\text { educational institutions across the globe, as well as the } \\
\text { ability to transfer credits by students. }\end{array}$ & \\
\hline 2. & $\begin{array}{l}\text { Organizational } \\
\text { Behaviour in } 21 \text { st } \\
\text { Century - 'Theory } \\
\text { A' for Managing } \\
\text { People for } \\
\text { Performance }\end{array}$ & $\begin{array}{l}\text { Theory X, Theory } \mathrm{Y} \text {, and subsequently Theory } \mathrm{Z} \text { arose } \\
\text { from postulates based on positive and negative } \\
\text { orientations to human nature. in each of these instances, } \\
\text { the fundamental 'desire for creativity in human nature was } \\
\text { ignored. according to ABCD analysis, the proposed } \\
\text { hypothesis emphasizes accountability as the dedication } \\
\text { that emerges from collective aim setting and assigning } \\
\text { responsibility rather than establishing targets and } \\
\text { accepting obligation. }\end{array}$ & [26] \\
\hline 3. & $\begin{array}{l}\text { Smart Library } \\
\text { Models for Future } \\
\text { Generations }\end{array}$ & $\begin{array}{l}\text { The development of information communication } \\
\text { technology has shown a new avenue for traditional library } \\
\text { models to innovate. countries can transform brick-and- } \\
\text { mortar libraries into click-and-mortar libraries, providing } \\
\text { rapid and comprehensive information to the general public } \\
\text { and students around the country and the world without } \\
\text { prejudice. the projected universal resource center is } \\
\text { intended to be a technological breakthrough that allows } \\
\text { any human being on the planet to exchange intangible } \\
\text { knowledge resources without barriers. }\end{array}$ & [27] \\
\hline 4. & Green Education & $\begin{array}{l}\text { There is a need for institutions to reassess how they } \\
\text { develop their syllabi. Innovative thinking is considered } \\
\text { necessary. The present regular structures and practices } \\
\text { should be disrupted, ushering in a new era of social } \\
\text { learning, one in which real-world problems and challenges } \\
\text { are identified and examined. a program that is appropriate } \\
\text { for people from various walks of life so that they may } \\
\text { participate in problem-solving that benefits people and the } \\
\text { environment. The tipping point will be instilling education } \\
\text { in young graduates and postgraduates so that they may } \\
\text { create careers as decision-makers in green education. In } \\
\text { today's world, traditional schooling is no longer sufficient. }\end{array}$ & [28] \\
\hline
\end{tabular}

\section{STRUCTURE OF ABCD ANALYSIS FRAMEWORK :}

Many Factors that affect the four identified constructs namely, Advantages, Benefits, Constraints, and Disadvantages, are taken from qualitative data and the constituent critical element were identified of Online Food Delivery Services [8], [29], [30].

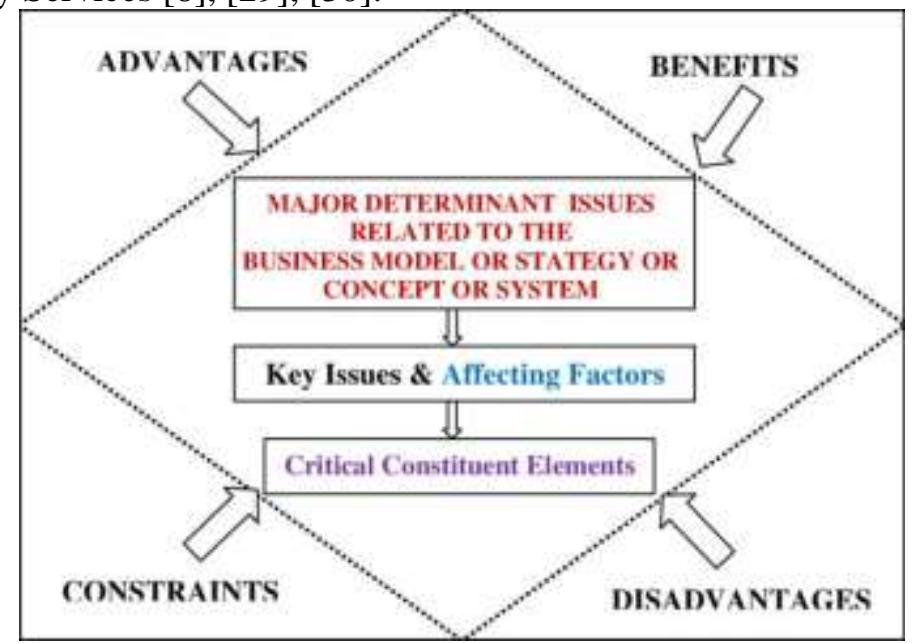

Fig. 1: Block Diagram indicating the factors affecting the Online Food Delivery Services as per ABCD Analysis Framework [6] 
7. KEY ATTRIBUTES AFFECTING ONLINE FOOD DELIVERY SERVICES :

Table 3: Exhibits of determinant issues and key attributes of Online Food Delivery Services

\begin{tabular}{|c|l|l|}
\hline Sl. No & \multicolumn{1}{|c|}{ Determinant Issues } & \multicolumn{1}{c|}{ Key Attributes } \\
\hline $\mathbf{1 .}$ & Supplier Issues (Restaurants) & Inventory, Competition, Health \& Hygiene \\
\hline $\mathbf{2 .}$ & Food Delivery Partner Issues & Responsiveness, Assurance, Marketing \\
\hline $\mathbf{3 .}$ & Customer Issues & Reliability, Convenience, Safety \\
\hline 4. & $\begin{array}{l}\text { Food Delivery Technology Issues } \\
\text { (Operating System) }\end{array}$ & Usage, Privacy, Payment \\
\hline Source: Compiled by the Researcher & \multicolumn{2}{l}{} \\
\hline
\end{tabular}

8. FACTOR ANALYSIS OF ONLINE FOOD DELIVERY SERVICES USING ABCD FRAMEWORK :

\begin{tabular}{|c|c|c|c|c|c|}
\hline \multicolumn{6}{|c|}{ Table 4: Exhibits of Factor Analysis of Online Food Delivery Services } \\
\hline $\begin{array}{c}\text { Determinan } \\
\text { t Issues }\end{array}$ & $\begin{array}{c}\text { Key } \\
\text { Attributes }\end{array}$ & Advantages & Benefits & Constraints & $\begin{array}{c}\text { Disadvantage } \\
\mathrm{s}\end{array}$ \\
\hline \multirow{3}{*}{$\begin{array}{c}\text { Supplier } \\
\text { (Restaurants) }\end{array}$} & Inventory & Fresh Stock & $\begin{array}{c}\text { EOQ } \\
\text { Benefits }\end{array}$ & $\begin{array}{l}\text { Shortage of } \\
\text { Supply }\end{array}$ & Blockage \\
\hline & Competition & $\begin{array}{c}\text { Increase } \\
\text { Uniqueness }\end{array}$ & $\begin{array}{l}\text { Differentiate } \\
\text { d Products }\end{array}$ & $\begin{array}{l}\text { Customer } \\
\text { Switch }\end{array}$ & Low Turnover \\
\hline & $\begin{array}{c}\text { Health \& } \\
\text { Hygiene }\end{array}$ & $\begin{array}{l}\text { Increase } \\
\text { Sales }\end{array}$ & $\begin{array}{l}\text { Increases } \\
\text { Demand }\end{array}$ & $\begin{array}{c}\text { Increased Food } \\
\text { Price }\end{array}$ & $\begin{array}{c}\text { High } \\
\text { Maintenance } \\
\text { Cost } \\
\end{array}$ \\
\hline \multirow{3}{*}{$\begin{array}{l}\text { Food } \\
\text { Delivery } \\
\text { Partner }\end{array}$} & $\begin{array}{c}\text { Responsivenes } \\
\text { s }\end{array}$ & $\begin{array}{l}\text { Order } \\
\text { Update }\end{array}$ & $\begin{array}{c}\text { Easy } \\
\text { Tracking }\end{array}$ & $\begin{array}{c}\text { High } \\
\text { Operational } \\
\text { Cost }\end{array}$ & $\begin{array}{c}\text { Cancellation of } \\
\text { Order }\end{array}$ \\
\hline & Assurance & $\begin{array}{c}\text { Safe } \\
\text { Delivery }\end{array}$ & $\begin{array}{c}\text { Reasonable } \\
\text { Price }\end{array}$ & Less Quantity & High Price \\
\hline & Marketing & $\begin{array}{l}\text { Widens } \\
\text { Customer } \\
\text { Base }\end{array}$ & $\begin{array}{c}\text { More } \\
\text { Outreach to } \\
\text { Untapped } \\
\text { Market }\end{array}$ & $\begin{array}{c}\text { Non- } \\
\text { Availability in } \\
\text { Rural Areas }\end{array}$ & $\begin{array}{l}\text { Cut -Throat } \\
\text { Competition }\end{array}$ \\
\hline \multirow{3}{*}{ Customer } & Reliability & $\begin{array}{l}\text { Affordable } \\
\text { Delivery }\end{array}$ & $\begin{array}{l}\text { Desired Food } \\
\text { Quality }\end{array}$ & $\begin{array}{l}\text { Wrong } \\
\text { Delivery }\end{array}$ & Brand Switch \\
\hline & Convenience & $\begin{array}{c}\text { Place } \\
\text { Convenienc } \\
\text { e }\end{array}$ & $\begin{array}{c}\text { Time } \\
\text { Convenience }\end{array}$ & High Traffic & Late Delivery \\
\hline & Safety & $\begin{array}{c}\text { Fresh } \\
\text { Ingredients }\end{array}$ & $\begin{array}{l}\text { Eco-Friendly } \\
\text { Packaging }\end{array}$ & $\begin{array}{c}\text { Lack of } \\
\text { Trustworthines } \\
\text { s of Deliverer }\end{array}$ & Low Morale \\
\hline \multirow{3}{*}{$\begin{array}{c}\text { Food } \\
\text { Delivery } \\
\text { Technology }\end{array}$} & Usage & $\begin{array}{c}\text { User- } \\
\text { Friendly } \\
\text { Interface }\end{array}$ & $\begin{array}{c}\text { Well- } \\
\text { Presented } \\
\text { Information }\end{array}$ & $\begin{array}{l}\text { Low Access to } \\
\text { The Internet }\end{array}$ & System Error \\
\hline & Privacy & $\begin{array}{c}\text { Data } \\
\text { Protection }\end{array}$ & $\begin{array}{l}\text { Increased } \\
\text { Usage }\end{array}$ & $\begin{array}{c}\text { Fear of } \\
\text { Personal Data } \\
\text { Theft } \\
\end{array}$ & Data Hacking \\
\hline & Payment & $\begin{array}{c}\text { Many } \\
\text { Modes of E- } \\
\text { Payment }\end{array}$ & $\begin{array}{l}\text { Easy E- } \\
\text { Payment }\end{array}$ & $\begin{array}{c}\text { Third-Party } \\
\text { Theft }\end{array}$ & No Credits \\
\hline
\end{tabular}

\section{ELEMENTARY ANALYSIS BASED ON CRITICAL CONSTITUENT ELEMENT :}

This section discusses in detail the various critical constituent element for each key attribute under the focus area belonging to the construct of the $\mathrm{ABCD}$ analysis framework. 
Table 5 below depicts Advantageous Factors of Online Food Delivery Services and their critical constituent element bought out by the Focus Group.

\begin{tabular}{|c|c|c|c|}
\hline $\begin{array}{l}\text { Determinant } \\
\text { Issues }\end{array}$ & Key Attributes & $\begin{array}{l}\text { Advantageous Factors } \\
\text { Affecting Determinant } \\
\text { Issues }\end{array}$ & Critical Constituent Elements \\
\hline \multirow{3}{*}{$\begin{array}{c}\text { Supplier } \\
\text { (Restaurants) }\end{array}$} & Inventory & Fresh Stock & High Working Capital \\
\hline & Competition & Increase Uniqueness & Increased Demand \\
\hline & $\begin{array}{l}\text { Health \& } \\
\text { Hygiene }\end{array}$ & Increase Sales & Increases Profits \\
\hline \multirow{3}{*}{$\begin{array}{l}\text { Food Delivery } \\
\text { Partner }\end{array}$} & Responsiveness & Order Update & Timely Delivery \\
\hline & Assurance & Safe Delivery & Repurchase \\
\hline & Marketing & Widens Customer Base & Brand Positioning \\
\hline \multirow{3}{*}{ Customer } & Reliability & Affordable Delivery & Often Orders \\
\hline & Convenience & Place Convenience & Economy \\
\hline & Safety & Fresh Ingredients & $\begin{array}{l}\text { Increase Customer Base of } \\
\text { Diet Conscious People }\end{array}$ \\
\hline \multirow{3}{*}{$\begin{array}{l}\text { Food Delivery } \\
\text { Technology }\end{array}$} & Usage & User-Friendly Interface & Ease of Use \\
\hline & Privacy & Data Protection & Confidence \\
\hline & Payment & Modes of E-Payment & Digital Payments \\
\hline
\end{tabular}

Table 6 given below depicts the Benefit Factors of Online Food Delivery Services and their critical constituent element bought out by the Focus Group.

Table 6: Exhibits of Benefit Factors of Online Food Delivery Services and their critical constituent elements

\begin{tabular}{|c|c|c|c|}
\hline $\begin{array}{l}\text { Determinant } \\
\text { Issues }\end{array}$ & Key Attributes & $\begin{array}{c}\text { Beneficial Factors } \\
\text { Affecting Determinant } \\
\text { Issues }\end{array}$ & $\begin{array}{l}\text { Critical Constituent } \\
\text { Elements }\end{array}$ \\
\hline \multirow{3}{*}{$\begin{array}{c}\text { Supplier } \\
\text { (Restaurants) }\end{array}$} & Inventory & EOQ Benefits & Balanced Stock Orders \\
\hline & Competition & Differentiated Products & High Capital Investment \\
\hline & $\begin{array}{l}\text { Health \& } \\
\text { Hygiene }\end{array}$ & Increases Demand & Government Restrictions \\
\hline \multirow{3}{*}{$\begin{array}{l}\text { Food Delivery } \\
\text { Partner }\end{array}$} & Responsiveness & Easy Tracking & Minimal Time \\
\hline & Assurance & Reasonable Price & Compromise on Quantity \\
\hline & Marketing & $\begin{array}{l}\text { More Outreach to } \\
\text { Untapped Market }\end{array}$ & Cultural Differences \\
\hline
\end{tabular}


International Journal of Case Studies in Business, IT, and Education (IJCSBE), ISSN: 2581-6942, Vol. 6, No. 1, February 2022

\begin{tabular}{|c|c|c|c|}
\hline \multirow{4}{*}{ Customer } & Reliability & Desired Food Quality & Price Skimming \\
\cline { 2 - 4 } & Convenience & Time Convenience & Reduced Lead Time \\
\cline { 2 - 4 } & Safety & Eco-Friendly Packaging & Health Safety \\
\hline \multirow{3}{*}{$\begin{array}{c}\text { Food Delivery } \\
\text { Technology }\end{array}$} & Usage & $\begin{array}{c}\text { Well-Presented } \\
\text { Information }\end{array}$ & More Clarity \\
\cline { 2 - 4 } & Privacy & Increased Usage & More Orders \\
\cline { 2 - 4 } & Payment & Easy E-Payment & More Promo Codes Savings \\
\hline
\end{tabular}

Source: Compiled by the Researcher

Table 7 given below depicts the Constraints Factors of Online Food Delivery Services and their critical constituent element bought out by the Focus Group.

\begin{tabular}{|c|c|c|c|}
\hline \multicolumn{4}{|c|}{ Table 7: Exhibits of Constraint Factors of Online Food Delivery Services and their critical } \\
constituent elements
\end{tabular}

Table 8 given below depicts the Disadvantageous Factors of Online Food Delivery Services and their critical constituent element bought out by the Focus Group. 
Table 8: Exhibits of Disadvantageous Factors of Online Food Delivery Services and their critical constituent elements

\begin{tabular}{|c|c|c|c|}
\hline $\begin{array}{l}\text { Determinant } \\
\text { Issues }\end{array}$ & Key Attributes & $\begin{array}{l}\text { Disadvantageous Factors } \\
\text { Affecting Determinant } \\
\text { Issues }\end{array}$ & $\begin{array}{l}\text { Critical Constituent } \\
\text { Elements }\end{array}$ \\
\hline \multirow{3}{*}{$\begin{array}{c}\text { Supplier } \\
\text { (Restaurants) }\end{array}$} & Inventory & Blockage & Order Cancellation \\
\hline & Competition & Low Turnover & Less Sales \\
\hline & $\begin{array}{c}\text { Health \& } \\
\text { Hygiene }\end{array}$ & High Maintenance Cost & $\begin{array}{c}\text { Less Orders from Low } \\
\text { Earners }\end{array}$ \\
\hline \multirow{3}{*}{$\begin{array}{l}\text { Food Delivery } \\
\text { Partner }\end{array}$} & Responsiveness & Cancellation of Order & Disappointment \\
\hline & Assurance & High Price & Occasional Orders \\
\hline & Marketing & Cut -Throat Competition & Short Term Survival \\
\hline \multirow{3}{*}{ Customer } & Reliability & Brand Switch & Reduced Customer Loyalty \\
\hline & Convenience & Late Delivery & Penalty \\
\hline & Safety & Low Morale & $\begin{array}{c}\text { Cautious Purchase } \\
\text { Intention }\end{array}$ \\
\hline \multirow{3}{*}{$\begin{array}{l}\text { Food Delivery } \\
\text { Technology }\end{array}$} & Usage & System Error & Orders Offline Mode \\
\hline & Privacy & Data Hacking & Diminished Reputation \\
\hline & Payment & No Credits & Cash Realisation \\
\hline & he Researcher & & \\
\hline
\end{tabular}

\section{QUANTITATIVE CRITICAL CONSTITUENT ELEMENT FOR ONLINE FOOD DELIVERY SERVICES AS PER ABCD ANALYSIS :}

The critical constituent element for each key attribute for Online Food Delivery under the determinant issues as per construct of $\mathrm{ABCD}$ analysis framework are outlined below:

Assignment of weights for each critical constituent element is done by the focus group. The weightage for the ranking is given as below:

$$
\begin{aligned}
& 3 \text { - Satisfactory } \\
& 2 \text { - Neutral } \\
& 1 \text { - Not Satisfactory }
\end{aligned}
$$

Table 9 given below depicts Advantageous Factors of Online Food Delivery Services and their critical constituent element bought out by the Focus Group.

\begin{tabular}{|c|c|c|c|c|c|c|}
\hline \multicolumn{6}{|c|}{ Table 9: Exhibits of Advantageous Factors of Online Food Delivery Services and their critical } \\
constituent element with the total mean score \\
\hline $\begin{array}{c}\text { Determinant } \\
\text { Issues }\end{array}$ & $\begin{array}{c}\text { Key } \\
\text { Attributes }\end{array}$ & $\begin{array}{c}\text { Advantageous } \\
\text { Factors } \\
\text { Affecting } \\
\text { Determinant } \\
\text { Issues }\end{array}$ & $\begin{array}{c}\text { Critical } \\
\text { Constituent } \\
\text { Elements }\end{array}$ & $\begin{array}{c}\text { Key } \\
\text { Attributes } \\
\text { (Total } \\
\text { Score) }\end{array}$ & $\begin{array}{c}\text { Determina } \\
\text { nt Issues } \\
\text { (Total } \\
\text { Score) }\end{array}$ & $\begin{array}{c}\text { Total } \\
\text { mean } \\
\text { score }\end{array}$ \\
\hline $\begin{array}{c}\text { Supplier } \\
\text { (Restaurants) }\end{array}$ & Inventory & Fresh Stock & $\begin{array}{c}\text { High } \\
\text { Working } \\
\text { Capital }\end{array}$ & 23 & 70 & $\mathbf{8 9 . 2}$ \\
\hline
\end{tabular}


International Journal of Case Studies in Business, IT, and Education

\begin{tabular}{|c|c|c|c|c|c|}
\hline & Competition & $\begin{array}{c}\text { Increase } \\
\text { Uniqueness }\end{array}$ & $\begin{array}{l}\text { Increased } \\
\text { Demand }\end{array}$ & 25 & \\
\hline & $\begin{array}{c}\text { Health \& } \\
\text { Hygiene }\end{array}$ & Increase Sales & $\begin{array}{l}\text { Increases } \\
\text { Profits }\end{array}$ & 22 & \\
\hline \multirow{3}{*}{$\begin{array}{c}\text { Food } \\
\text { Delivery } \\
\text { Partner }\end{array}$} & $\begin{array}{c}\text { Responsivene } \\
\text { ss }\end{array}$ & Order Update & $\begin{array}{c}\text { Timely } \\
\text { Delivery }\end{array}$ & 26 & \multirow{3}{*}{75} \\
\hline & Assurance & Safe Delivery & Repurchase & 23 & \\
\hline & Marketing & $\begin{array}{c}\text { Widens } \\
\text { Customer Base }\end{array}$ & $\begin{array}{c}\text { Brand } \\
\text { Positioning }\end{array}$ & 26 & \\
\hline \multirow[b]{3}{*}{ Customer } & Reliability & $\begin{array}{c}\text { Affordable } \\
\text { Delivery }\end{array}$ & $\begin{array}{l}\text { Often } \\
\text { Orders }\end{array}$ & 26 & \multirow[b]{3}{*}{74} \\
\hline & Convenience & $\begin{array}{c}\text { Place } \\
\text { Convenience }\end{array}$ & Economy & 25 & \\
\hline & Safety & $\begin{array}{c}\text { Fresh } \\
\text { Ingredients }\end{array}$ & $\begin{array}{c}\text { Increase } \\
\text { Customer } \\
\text { Base of Diet } \\
\text { Conscious } \\
\text { People }\end{array}$ & 23 & \\
\hline \multirow{3}{*}{$\begin{array}{c}\text { Food } \\
\text { Delivery } \\
\text { Technology }\end{array}$} & Usage & $\begin{array}{l}\text { User-Friendly } \\
\text { Interface }\end{array}$ & Ease of Use & 25 & \multirow{3}{*}{76} \\
\hline & Privacy & $\begin{array}{c}\text { Data } \\
\text { Protection }\end{array}$ & Confidence & 27 & \\
\hline & Payment & $\begin{array}{l}\text { Many Modes } \\
\text { of E-Payment }\end{array}$ & $\begin{array}{c}\text { More } \\
\text { Digital } \\
\text { Payments }\end{array}$ & 24 & \\
\hline
\end{tabular}

Table 10 given below depicts Benefit Factors of Online Food Delivery Services and their critical constituent element bought out by the Focus Group.

\begin{tabular}{|c|c|c|c|c|c|c|}
\hline \multicolumn{7}{|c|}{$\begin{array}{c}\text { Table 10: Exhibits of Benefit Factors of Online Food Delivery Services and their critical constituent } \\
\text { element with the total mean score }\end{array}$} \\
\hline $\begin{array}{l}\text { Determinan } \\
\text { t Issues }\end{array}$ & $\begin{array}{c}\text { Key } \\
\text { Attributes }\end{array}$ & $\begin{array}{c}\text { Beneficial } \\
\text { Factors } \\
\text { Affecting } \\
\text { Determinant } \\
\text { Issues } \\
\end{array}$ & $\begin{array}{c}\text { Critical } \\
\text { Constituent } \\
\text { Elements }\end{array}$ & $\begin{array}{c}\text { Key } \\
\text { Attribute } \\
\text { s (Total } \\
\text { Score) }\end{array}$ & $\begin{array}{c}\text { Determina } \\
\text { nt Issues } \\
\text { (Total } \\
\text { Score) }\end{array}$ & $\begin{array}{l}\text { Total } \\
\text { mean } \\
\text { score }\end{array}$ \\
\hline \multirow{3}{*}{$\begin{array}{c}\text { Supplier } \\
\text { (Restaurants) }\end{array}$} & Inventory & EOQ Benefits & $\begin{array}{c}\text { Balanced } \\
\text { Stock Orders }\end{array}$ & 22 & \multirow{3}{*}{72} & \multirow{7}{*}{88.5} \\
\hline & Competition & $\begin{array}{l}\text { Differentiated } \\
\text { Products }\end{array}$ & $\begin{array}{l}\text { High Capital } \\
\text { Investment }\end{array}$ & 27 & & \\
\hline & $\begin{array}{l}\text { Health \& } \\
\text { Hygiene }\end{array}$ & $\begin{array}{c}\text { Increases } \\
\text { Demand }\end{array}$ & $\begin{array}{l}\text { Government } \\
\text { Restrictions }\end{array}$ & 23 & & \\
\hline \multirow{3}{*}{$\begin{array}{l}\text { Food } \\
\text { Delivery } \\
\text { Partner }\end{array}$} & $\begin{array}{l}\text { Responsiven } \\
\text { ess }\end{array}$ & Easy Tracking & $\begin{array}{l}\text { Minimal } \\
\text { Time }\end{array}$ & 25 & \multirow{3}{*}{75} & \\
\hline & Assurance & $\begin{array}{l}\text { Reasonable } \\
\text { Price }\end{array}$ & $\begin{array}{l}\text { Compromise } \\
\text { on Quantity }\end{array}$ & 24 & & \\
\hline & Marketing & $\begin{array}{l}\text { More Outreach } \\
\text { to Untapped } \\
\text { Market }\end{array}$ & $\begin{array}{l}\text { Cultural } \\
\text { Differences }\end{array}$ & 26 & & \\
\hline Customer & Reliability & $\begin{array}{l}\text { Desired Food } \\
\text { Quality }\end{array}$ & $\begin{array}{c}\text { Price } \\
\text { Skimming }\end{array}$ & 27 & 75 & \\
\hline
\end{tabular}


International Journal of Case Studies in Business, IT, and Education

\begin{tabular}{|c|c|c|c|c|c|}
\hline & Convenience & $\begin{array}{c}\text { Time } \\
\text { Convenience }\end{array}$ & $\begin{array}{c}\text { Reduced } \\
\text { Lead Time }\end{array}$ & 27 & \\
\hline & Safety & $\begin{array}{c}\text { Eco-Friendly } \\
\text { Packaging }\end{array}$ & Health Safety & 21 & \\
\hline \multirow{3}{*}{$\begin{array}{c}\text { Food } \\
\text { Delivery } \\
\text { Technology }\end{array}$} & Usage & $\begin{array}{l}\text { Well-Presented } \\
\text { Information }\end{array}$ & More Clarity & 26 & \multirow{3}{*}{75} \\
\hline & Privacy & Increased Usage & More Orders & 23 & \\
\hline & Payment & $\begin{array}{l}\text { Easy E- } \\
\text { Payment }\end{array}$ & $\begin{array}{l}\text { More Promo } \\
\text { Codes } \\
\text { Savings }\end{array}$ & 26 & \\
\hline
\end{tabular}

Table 11 given below depicts Constraints Factors of Online Food Delivery Services and their critical constituent element bought out by the Focus Group.

\begin{tabular}{|c|c|c|c|c|c|c|}
\hline $\begin{array}{l}\text { Determinant } \\
\text { Issues }\end{array}$ & $\begin{array}{c}\text { Key } \\
\text { Attributes }\end{array}$ & $\begin{array}{l}\text { Constraints } \\
\text { Factors } \\
\text { Affecting } \\
\text { Determinant } \\
\text { Issues } \\
\end{array}$ & $\begin{array}{c}\text { Critical } \\
\text { Constituent } \\
\text { Elements }\end{array}$ & $\begin{array}{c}\text { Key } \\
\text { Attribute } \\
\text { s (Total } \\
\text { Score) }\end{array}$ & $\begin{array}{c}\text { Determina } \\
\text { nt Issues } \\
\text { (Total } \\
\text { Score) }\end{array}$ & $\begin{array}{l}\text { Total } \\
\text { mean } \\
\text { score }\end{array}$ \\
\hline \multirow{3}{*}{$\begin{array}{c}\text { Supplier } \\
\text { (Restaurants) }\end{array}$} & Inventory & $\begin{array}{l}\text { Shortage of } \\
\text { Supply }\end{array}$ & Hiked Price & 23 & \multirow{3}{*}{70} & \multirow{11}{*}{84.5} \\
\hline & Competition & $\begin{array}{l}\text { Customer } \\
\text { Switch }\end{array}$ & $\begin{array}{c}\text { High } \\
\text { Customer } \\
\text { Retention } \\
\text { Cost }\end{array}$ & 23 & & \\
\hline & $\begin{array}{c}\text { Health \& } \\
\text { Hygiene }\end{array}$ & $\begin{array}{l}\text { Increased } \\
\text { Food Price }\end{array}$ & $\begin{array}{c}\text { High } \\
\text { Maintenance } \\
\text { Cost } \\
\end{array}$ & 24 & & \\
\hline \multirow{3}{*}{$\begin{array}{c}\text { Food } \\
\text { Delivery } \\
\text { Partner }\end{array}$} & $\begin{array}{c}\text { Responsivenes } \\
\text { s }\end{array}$ & $\begin{array}{c}\text { High } \\
\text { Operational } \\
\text { Cost }\end{array}$ & Price Hiking & 24 & \multirow{3}{*}{70} & \\
\hline & Assurance & Less Quantity & $\begin{array}{l}\text { Increased } \\
\text { Economy }\end{array}$ & 23 & & \\
\hline & Marketing & $\begin{array}{c}\text { Non- } \\
\text { Availability in } \\
\text { Rural Areas }\end{array}$ & $\begin{array}{c}\text { Low } \\
\text { Standard of } \\
\text { Living }\end{array}$ & 23 & & \\
\hline \multirow{3}{*}{ Customer } & Reliability & $\begin{array}{c}\text { Wrong } \\
\text { Delivery }\end{array}$ & $\begin{array}{c}\text { Brand } \\
\text { Switch }\end{array}$ & 24 & \multirow{3}{*}{71} & \\
\hline & Convenience & High Traffic & $\begin{array}{c}\text { Late } \\
\text { Delivery }\end{array}$ & 25 & & \\
\hline & Safety & $\begin{array}{c}\text { Lack of } \\
\text { Trustworthine } \\
\text { ss of Deliverer }\end{array}$ & $\begin{array}{l}\text { More Dine } \\
\text { Out }\end{array}$ & 22 & & \\
\hline \multirow{2}{*}{$\begin{array}{c}\text { Food } \\
\text { Delivery } \\
\text { Technology }\end{array}$} & Usage & $\begin{array}{l}\text { Low Access to } \\
\text { the Internet }\end{array}$ & $\begin{array}{c}\text { Less } \\
\text { Informed }\end{array}$ & 25 & \multirow[b]{2}{*}{70} & \\
\hline & Privacy & $\begin{array}{c}\text { Fear of } \\
\text { Personal Data } \\
\text { Theft } \\
\end{array}$ & $\begin{array}{l}\text { More Tele- } \\
\text { Orders }\end{array}$ & 25 & & \\
\hline
\end{tabular}


International Journal of Case Studies in Business, IT, and Education

\begin{tabular}{|l|c|c|c|c|c|c|}
\hline & Payment & $\begin{array}{c}\text { Third-Party } \\
\text { Theft }\end{array}$ & $\begin{array}{c}\text { More Cash } \\
\text { Transactions }\end{array}$ & 20 & & \\
\hline
\end{tabular}

Table 12 given below depicts Disadvantageous Factors of Online Food Delivery Services and their critical constituent element bought out by the Focus Group.

\begin{tabular}{|c|c|c|c|c|c|c|}
\hline $\begin{array}{l}\text { Determinant } \\
\text { Issues }\end{array}$ & $\begin{array}{c}\text { Key } \\
\text { Attributes }\end{array}$ & $\begin{array}{c}\text { Disadvantageo } \\
\text { us Factors } \\
\text { Affecting } \\
\text { Determinant } \\
\text { Issues }\end{array}$ & $\begin{array}{c}\text { Critical } \\
\text { Constituent } \\
\text { Elements }\end{array}$ & $\begin{array}{c}\text { Key } \\
\text { Attribu } \\
\text { tes } \\
\text { (Total } \\
\text { Score) }\end{array}$ & $\begin{array}{c}\text { Determi } \\
\text { nant } \\
\text { Issues } \\
\text { (Total } \\
\text { Score) }\end{array}$ & $\begin{array}{l}\text { Total } \\
\text { mean } \\
\text { score }\end{array}$ \\
\hline \multirow{3}{*}{$\begin{array}{c}\text { Supplier } \\
\text { (Restaurants) }\end{array}$} & Inventory & Blockage & $\begin{array}{c}\text { Order } \\
\text { Cancellation }\end{array}$ & 22 & \multirow{3}{*}{71} & \multirow{12}{*}{79.2} \\
\hline & Competition & Low Turnover & Less Sales & 25 & & \\
\hline & $\begin{array}{c}\text { Health \& } \\
\text { Hygiene }\end{array}$ & $\begin{array}{c}\text { High } \\
\text { Maintenance } \\
\text { Cost }\end{array}$ & $\begin{array}{l}\text { Less Orders } \\
\text { from Average } \\
\text { and Low } \\
\text { Earners }\end{array}$ & 24 & & \\
\hline \multirow{3}{*}{$\begin{array}{c}\text { Food } \\
\text { Delivery } \\
\text { Partner }\end{array}$} & $\begin{array}{c}\text { Responsiven } \\
\text { ess }\end{array}$ & $\begin{array}{c}\text { Cancellation Of } \\
\text { Order }\end{array}$ & $\begin{array}{c}\text { Disappointme } \\
\mathrm{nt}\end{array}$ & 24 & \multirow{3}{*}{71} & \\
\hline & Assurance & High Price & $\begin{array}{c}\text { Occasional } \\
\text { Orders }\end{array}$ & 22 & & \\
\hline & Marketing & $\begin{array}{l}\text { Cut -Throat } \\
\text { Competition }\end{array}$ & $\begin{array}{c}\text { Short Term } \\
\text { Survival }\end{array}$ & 25 & & \\
\hline \multirow{3}{*}{ Customer } & Reliability & Brand Switch & $\begin{array}{c}\text { Reduced } \\
\text { Customer } \\
\text { Loyalty }\end{array}$ & 21 & \multirow{3}{*}{65} & \\
\hline & Convenience & Late Delivery & Penalty & 23 & & \\
\hline & Safety & Low Morale & $\begin{array}{l}\text { Cautious } \\
\text { Purchase } \\
\text { Intention } \\
\end{array}$ & 21 & & \\
\hline \multirow{3}{*}{$\begin{array}{c}\text { Food } \\
\text { Delivery } \\
\text { Technology }\end{array}$} & Usage & System Error & $\begin{array}{c}\text { Orders } \\
\text { Offline Mode }\end{array}$ & 24 & \multirow{3}{*}{75} & \\
\hline & Privacy & Data Hacking & $\begin{array}{l}\text { Diminished } \\
\text { Reputation }\end{array}$ & 26 & & \\
\hline & Payment & No Credits & $\begin{array}{c}\text { Cash } \\
\text { Realisation }\end{array}$ & 25 & & \\
\hline
\end{tabular}

\section{GRAPHICAL REPRESENTATION OF AFFECTING FACTORS FOR ONLINE FOOD DELIVERY SERVICES AS PER ABCD ANALYSIS FRAMEWORK :}

The total mean score value of Online Food Delivery to understand the major affecting factor as per $\mathrm{ABCD}$ analysis framework is given below in Figure 2. 


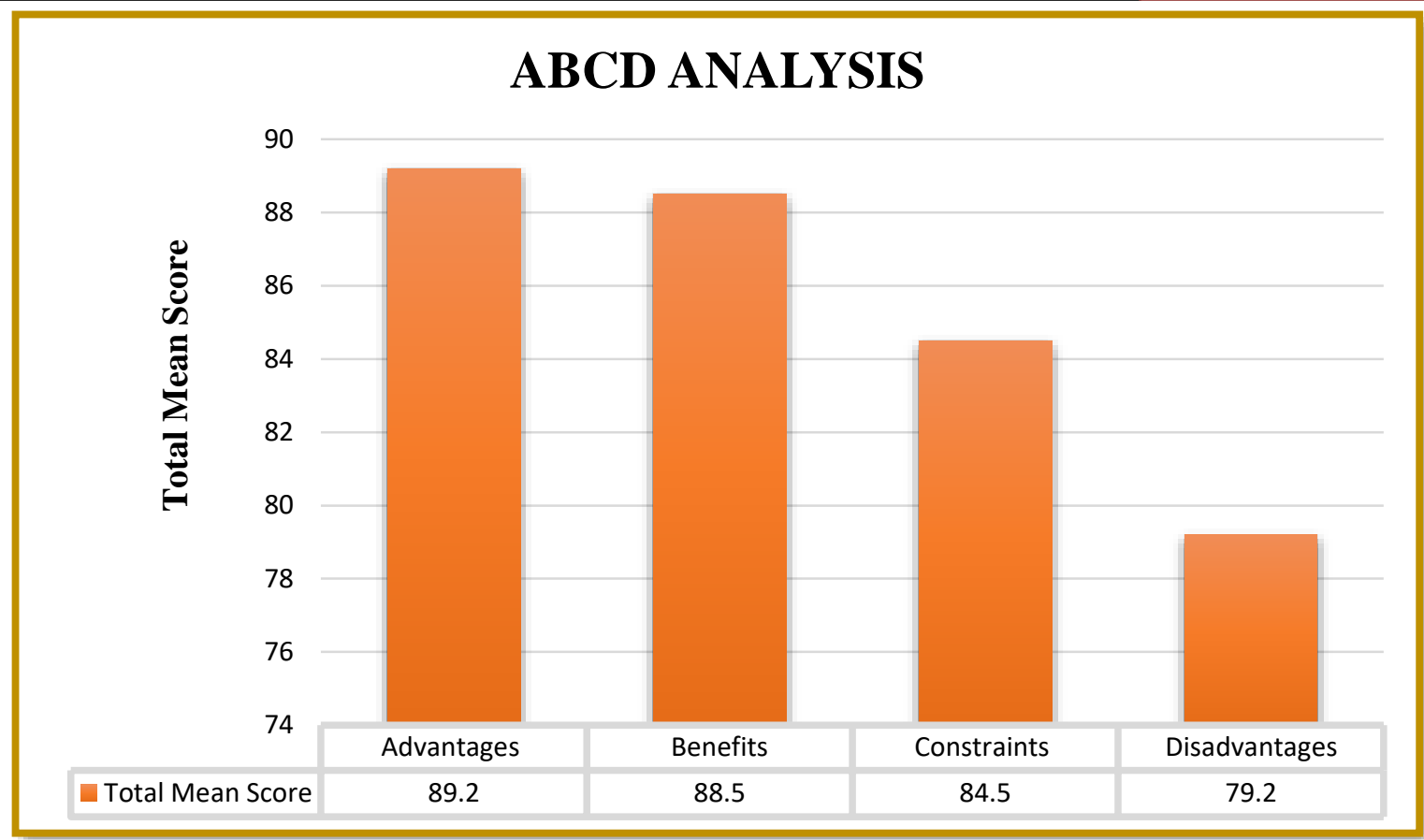

Fig. 2: Exhibits of Total Mean Score of Online Food Delivery Services as per ABCD analysis framework

\section{Interpretation:}

The above figure depicts that affecting factors for Online Food Delivery Services as per ABCD analysis, one - Advantages factor scores highest with total mean score value of 89.2 , which means it is satisfactory. Second - Benefits with total the mean score value as 88.5, which means it is neutral affecting factor. Third- Constraints and Disadvantages factor with total mean score value as 84.5 and 79.2, which depicts those factors are not satisfactory for the Online Food Delivery Services business model.

\section{CONCLUSION :}

$\mathrm{ABCD}$ analysis framework is a comprehensive model which helps in analyzing different business models, strategy, concepts or system. The study used the ABCD analysis framework to determine the affecting factors on Online Food Delivery Services and found Advantageous factors were major contributors to its success. The research study strongly argues in favour of ABCD analysis framework for assessing other business models, strategy, concepts or system.

\section{REFERENCES :}

[1] Frederick, D. P., \& Parappagoudar, S. K. (2021). A Descriptive Analysis on Sustainable Business Strategy of Online Food Service Industry. International Research Journal of Modernization in Engineering Technology and Science, 3(3), 545-554.

Google Scholar $X^{7}$

[2] Frederick, D. P., Nayak, P., \& Reddy, B. (2021). E-Business Processes in Food Services. International Journal of Advanced Research, 9(10), 821-829.

Google Scholar $x^{7}$

[3] Frederick, D. P., \& Bhat, G. (2021). Review on Customer Perception Towards Online Food Delivery Services. International Journal of Creative Research Thoughts, 9(7), b301-b314.

Google Scholar $X^{7}$

[4] Purvis, B., Mao, Y., \& Robinson, D. (2019). Three pillars of sustainability: in search of conceptual origins. Sustainability science, 14(3), 681-695.

Google Scholar $\nearrow^{\top}$ 
[5] Frederick, D. P., \& Parappagoudar, S. K. (2021). SWOC Analysis of Zomato-A Case of Online Food Delivery Services. International Research Journal of Modernization in Engineering Technology and Science, 3(3),537-544.

Google Scholar $x^{7}$

[6] Aithal, P. S., Shailashree, V., \& Kumar, P. M. (2015). A new ABCD technique to analyse business models \& concepts. International Journal of Management, IT and Engineering, 5(4), 409-423. Google Scholar X

[7] Aithal, P. S. (2017). Industry Analysis-The First Step in Business Management Scholarly Research. International Journal of Case Studies in Business, IT and Education (IJCSBE), 1(1), 113.

Google Scholar X

[8] Aithal, P. S., Shailashree, V., \& Kumar, P. M. (2015). Application of ABCD Analysis Model for Black Ocean Strategy. International Journal of Applied Research, 1(10), 331-337. Google Scholar X'

[9] Aithal, P. S., Shailashree, V., \& Kumar, P. M. (2016). Analysis of NAAC Accreditation System using ABCD framework. International Journal of Management, IT and Engineering, 6(1), 30-44. Google Scholar $X^{\top}$

[10] Aithal, P. S., Shailashree, V., \& Kumar, P. M. (2016). Analysis of NAAC Accreditation System using ABCD framework. International Journal of Management, IT and Engineering, 6(1), 30-44. Google Scholar X

[11] Aithal, P. S., Shailashree, V., \& Kumar, P. M. (2016). Application of ABCD Analysis Framework on Private University System in India. International Journal of Management Sciences and Business Research, 5(4), 159-170.

Google Scholar X

[12] Reshma, P. S., Acharya, S., \& Aithal, P. S. (2015). Relevance of On-line Office Administration through Working from Home in Future Education System. International Journal of Application or Innovation in Engineering \& Management (IJAIEM), 4(4), 44-53.

Google Scholar $\chi^{\top}$

[13] Reshma, P. S., Aithal, P. S., \& Acharya, S. (2015). An empirical study on Working from Home: A popular e-business model. International Journal of Advance and Innovative Research, 2(2), 12 18 .

Google Scholar $\chi^{7}$

[14] Aithal, P. S., \& Suresh Kumar, P. M. (2015). Enhancement of Graduate attributes in Higher Education Institutions through Stage Models. IMPACT: International Journal of Research in Business Management, 3(3), 121-130.

Google Scholar $X^{\top}$

[15] Aithal, P. S., \& Kumar, P. M. (2015). Black Ocean Strategy-A Probe into a new type of Strategy used for Organizational Success. GE-International Journal of Management Research (GEIJMR), 3(8), 45-65.

Google Scholar ${ }^{7}$

[16] Muduli, A. K., Pati, P. K., \& Pattnaik, M. Application of ABCD Analysis Framework on Private University System in India. IJEMHS, 31(3),131-135.

Google Scholar $x^{\top}$

[17] Aithal, P. S., \& Pai T, V. (2016). Concept of Ideal Software and its Realization Scenarios. International Journal of Scientific Research and Modern Education (IJSRME), I(1), 826-837. 
Google Scholar X

[18] Aithal, P. S., VT, S., \& Kumar, P. M. (2016). Analysis of ABC Model of Annual Research Productivity using ABCD Framework. International Journal of Current Research and Modern Education (IJCRME), 1(1), 846-858.

Google Scholar $\nearrow^{\top}$

[19] Aithal, S., \& Aithal, P. S. (2016). ABCD analysis of Dye-doped Polymers for Photonic Applications. IRA-International Journal of Applied Sciences, 4(3), 358-378.

Google Scholar X

[20] Aithal, P. S., Kumar, P. M., \& Shailashree, V. (2016). Factors \& elemental analysis of six thinking hats technique using ABCD framework IRA-International Journal of Applied Sciences, 1(1),8595 .

\section{Google Scholar $x^{\lambda}$}

[21] Aithal, P. S., \& Aithal, S. (2017). Factor Analysis based on ABCD Framework on Recently Announced New Research Indices. International Journal of Management, Technology, and Social Sciences (IJMTS), 1(1), 82-94.

Google Scholar ${ }^{\top}$

[22] Aithal, A., \& Aithal, P. S. (2017). ABCD analysis of task shifting-an optimum alternative solution to professional healthcare personnel shortage. International Journal of Health Sciences and Pharmacy (IJHSP), 1(2), 36-51.

Google Scholar $X^{\top}$

[23] Shenoy, V., \& Aithal, P. S. (2017). Quantitative ABCD Analysis of IEDRA Model of Placement Determination. International Journal of Case Studies in Business, IT and Education (IJCSBE), 1(2), 103-113.

Google Scholar $X^{\top}$

[24] Raj, K., \& Aithal, P. S. (2018). Generating Wealth at the Base of the Pyramid-a Study Using ABCD Analysis Technique. International Journal of Computational Research and Development (IJCRD), 3(1), 68-76.

Google Scholar $7^{-76}$

[25] Aithal, P. S., \& Kumar, P. M. (2016). Analysis of Choice Based Credit System in Higher Education. International Journal of Engineering Research and Modern Education (IJERME), I(I), 278-284.

Google Scholar $x^{7}$

[26] Aithal, P. S., \& Kumar, P. M. (2016). Organizational behaviour in 21st century- 'Theory A' for managing people for performance. IOSR Journal of Business and Management (IOSRJBM), 18(7), 126-134.

Google Scholar $x^{\top}$

[27] Aithal, P. S. (2016). Smart Library Model for Future Generations. International Journal of Engineering Research and Modern Education (IJERME), 1(1), 693-703.

Google Scholar $X^{\top}$

[28] Aithal, P. S., \& Rao, P. (2016). Green education concepts \& strategies in higher education model. International Journal of Scientific Research and Modern Education (IJSRME), 1(1),793802.

Google Scholar $x^{7}$

[29] Rogers, E. M. (1995). Diffusion of Innovation: modifications of a model for telecommunications. In Die diffusion von innovationen in der telekommunikation (pp. 25-38). Springer, Berlin, Heidelberg. 
Google Scholar $X^{\top}$

[30] Morgan, R. M., \& Hunt, S. D. (1994). The commitment-trust theory of relationship marketing. Journal of marketing, 58(3), 20-38.

Google Scholar ${ }^{\top}$ 